\title{
Heterocyclization Reaction of 4-(2-Methylaziridin-1-yl)-3-ureidobenzotrifluorides under Appel's Conditions
}

\author{
Hyun In Cho and Kee-Jung Lee ${ }^{\circ}$ \\ Department of Chemical Engineering, Hanang Lniversitv, Seoul 133-791, Korea \\ Received October 28, 2002
}

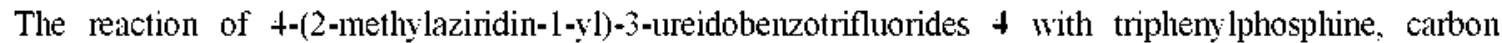
tetrachloride, and triethylamine (Appel's condition) led to the corresponding carbodiimides $\mathbf{5}$. which undenvent intramolecular cycloaddition reaction with aziridine under the reaction condition to give the benzinidazolefused heterocycles. 2.3-dilydro- $1 H$-imidazo[1.2-a]benzimidazoles 8 and 12.13 -dihydro- $5 H$-benzimidazo[2.3- $b]$ [1,3]benzodiazepines 9 .
\end{abstract}

Key Words : Heterocyclization. Urea. Aziridine, Carbodiimide. Appel's conditions

\section{Introduction}

2.3-Dihydroimidazo[ $1.2-a]$ benzimidazole derivatives have been shown to exhibit antihypertensive. ${ }^{l}$ antihistamine. antidiabetic. ${ }^{3}$ and antiarrhythmic ${ }^{3}$ activities and this justifies continuous efforts in developing more general and versatile synthetic methodologies to this class of compounds. Usually they are prepared by the intramolecular substitution reaction of suitable functionalized benzimidazoles having amine moieties. ${ }^{+}$

We have recently reported a new synthesis of 5.6-dihydro$7 \mathrm{H}$-imidazo[1,2-b][1,2.4]triazoles. ${ }^{5}$ 2.3-dihydro- $1 \mathrm{H}$-imidazo$\left[2^{\prime}, 3^{\prime}: 2.3\right]$ imidazo[4.5-b]py'ridines $s^{6}$ and 12.13-dihydro-5 $H^{-}$ 1.3-benzodiazepino $\left[2^{\prime} \cdot 3^{\prime}: 2.3\right]$ imidazo $[4.5-b]$ py ridines $s^{6}$ involving intramolecular cycloaddition reaction of aziridine and carbodiimide ${ }^{7}$ obtained from the corresponding ureas using Appel's dehydration condition ${ }^{8}$ as shown in Scheme 1 and Scheme 2. The present paper describes the synthesis of related heterocycles. 2.3-dihy'dro- $1 H$-imidazo[1,2-a]benzimidazoles 8 and hitherto unknown 12.13-dihydro-5 $\mathrm{H}$ -

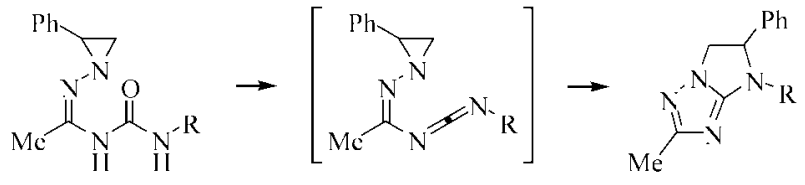

Scheme 1

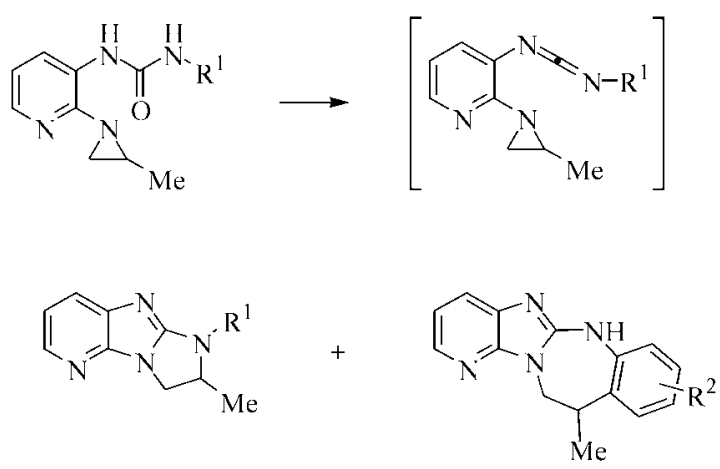

Scheme 2 benzimidazo[2.3-b][1.3]benzodiazepines 9 by the similar manner (Scheme 3).

\section{Results and Discussion}

The four-step synthetic approach to 8 and 9 required linking an aziridine moiety to a phenyl ring followed by reduction of the nitro group. urea formation and cyclization under Appel's condition to the benzimidazole-fused heterocylces 8 and 9 as shown in Scheme 3. Thus. 4-chloro-3nitrobenzotrifluoride (1) was reacted with 2-methylaziridine in the presence of triethylamine in tetrahydrofuran at $60^{\circ} \mathrm{C}$ for $48 \mathrm{~h}$ to give 4-(2-methylaziridin-1-yl)-3-nitrobenzotrifluoride (2) in $93 \%$ yield. Reduction of the nitro group of 2 with $5 \%$ palladium on charcoal in hydrazine hydrate at room temperature for $2 \mathrm{~h}$ afforded aniline 3 in $91 \%$ yield. Treatment of the aniline 3 with isocyanates in dichloromethane at room temperature gave the ureas + in $86-91 \%$ yields. Reaction of ureas 4 with triphenylphosphine, carbon tetrachloride and triethylamine in refluxing dichloromethane for $2-24 \mathrm{~h}$ afforded 2.3 -dihydro- $H$-imidazo[1.2- $a]$ benzimidazoles $8(54-78 \%)$ as a major product and 12.13dihydro-5 $H$-benzimidazo[2.3- $b][1.3]$ benzodiazepines 9 (4$8 \%$ ) as a minor one. In the case of $N$-methyl urea te. a single product $8 \mathbf{e}(74 \%)$ was isolated as a $\mathrm{HCl}$ salt.

A suitable mechanism for the formation of 8 and 9 is depicted in Scheme 3. Although the isolation of carbodiimides 5 was unsuccessful under the reaction conditions. an intramolecular cycloaddition reaction of aziridinyl carbodiimides 5 may give the zwitterionic aziridinium ions 6 followed by aziridine ring opening to afford the resonancestabilized zwitterionic intermediates $7 \mathbf{a}-\mathbf{b}$ and subsequent ring closure to give $\mathbf{8}$ and $\mathbf{9}$ after rearomatization. Presumably the low yields of the minor products 9 might be explained by the small contribution of resonance form $7 \mathrm{~b}$ due to the loss of resonance energy of benzene ring.

The structures 8 and 9 were assigned on the basis of spectroscopic data. Compound 8a. for instance, had the molecular formula of $\mathrm{C}_{17} \mathrm{H}_{14} \mathrm{~F}_{3} \mathrm{~N}_{3}$. as indicated by mass spectra $\left(\mathrm{M}^{+} 317\right)$ as a base peak. In the ${ }^{1} \mathrm{H}$ NMR spectrum of 

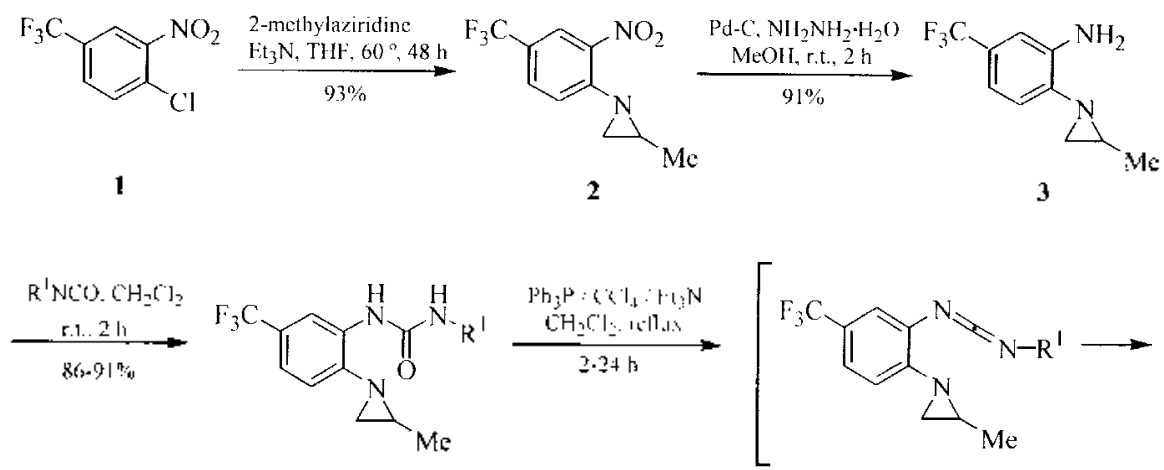

4a, $\mathrm{R}^{\prime}=\mathrm{C}_{6} \mathrm{H}_{5} \quad 4 \mathrm{~d}, \mathrm{R}^{\prime}=p-\mathrm{MeOC}_{6} \mathrm{H}_{4} \quad 5$

4b, $\mathrm{R}^{\mathrm{l}}=p-\mathrm{ClC}_{6} \mathrm{H}_{4} \quad$ 4e, $\mathrm{R}^{1}=\mathrm{Me}$

$4 \mathrm{c}, \mathrm{R}^{\prime}=0 \cdot \mathrm{FC}_{6} \mathrm{H}_{4}$

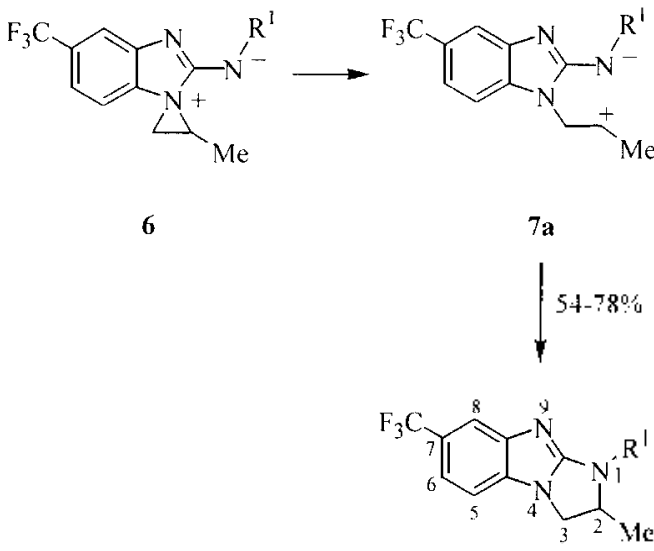

8

$$
\begin{aligned}
& \text { 8a: } R^{1}=C_{6} H_{5} \\
& \text { 8b, } \mathrm{R}^{\mathrm{l}}=p-\mathrm{ClC}_{6} \mathrm{H}_{4} \\
& \text { 8c. } R^{\prime}={ }^{\prime}-\mathrm{FC}_{4} \mathrm{ll}_{4} \\
& \text { 8d, } R^{\prime}=p-M_{e} \mathrm{OC}_{6} \mathrm{H}_{4} \\
& \text { 8e. } R^{\prime}=\mathrm{Me}
\end{aligned}
$$

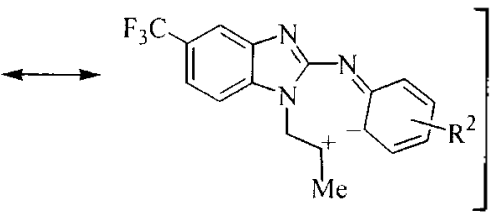

$7 \mathbf{b}$
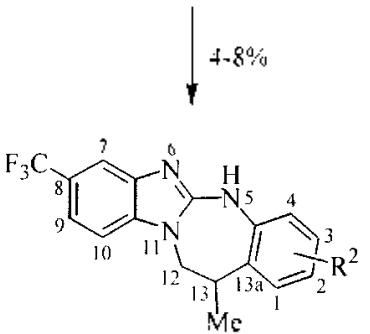

9

9a. $\mathrm{R}^{2}=\mathrm{H}$

$9 \mathbf{h}, \mathrm{R}^{2}=p-\mathrm{Cl}$

9c, $\mathrm{R}^{2}=\vartheta-\mathrm{F}$

9d. $R^{?}=p \cdot M e O$

Scheme 3

8a. the signals from the two C3 hydrogens appear as two doublet of doublets at $4.26(J=15.6$ and $7.3 \mathrm{~Hz})$ and 4.36 $\operatorname{ppm}(J=15.6$ and $4.5 \mathrm{~Hz})$. which arise as a result of coupling of the nonequivalent geminal hydrogens with one another and of each of them with the $\mathrm{C} 2$ hydrogen. The signal corresponding to the $\mathrm{C} 2$ hydrogen appears as a complex multiplet at $4.48 \mathrm{ppm}$. which arises from coupling with the $\mathrm{C} 3$ hydrogen atoms and the $\mathrm{C} 2$ methyl group. The ${ }^{13} \mathrm{C}$ NMR showed fifteen absorption peaks and its infrared spectrum showed no absorption in the region near $3400 \mathrm{~cm}^{-1}$. Compound 9a had the molecular formula of $\mathrm{C}_{17} \mathrm{H}_{14} \mathrm{~F}_{3} \mathrm{~N}_{3}$. as indicated by mass spectra $\left(\mathrm{M}^{-} 317\right)$ as a base peak again. Comparison of the ${ }^{l} \mathrm{H}$ NMR signals for the $\mathrm{CH}_{2}(\delta 4.03, \mathrm{dd}$. $J=9.5$ and $5.8 \mathrm{~Hz}$ and $\delta 4.60$.dd. $J=9.5$ and $8.9 \mathrm{~Hz}$ ) and $\mathrm{CH}(\delta 4.76 . \mathrm{m})$ groups with those of $8 \mathbf{a}$ showed different coupling constant (15.6 vs 9.5). Unfortunately no N-H proton was observed distinctly. The ${ }^{13} \mathrm{C}$ NMR exhibited seventeen absorption peaks including peak at $\delta=133.0$ assignable to the bridged carbon (C13a). and its infrared spectrum showed absorption for $\mathrm{NH}$ band $\left(3409 \mathrm{~cm}^{-1}\right)$.

In conclusion. ușing 4-(2-methỵlaziridin-1-yl)-3-ureidobenzotrifluoride 4 in the new synthesis of benzinidazolefused heterocycles via intramolecular cycloaddition reaction under Appel's conditions was achieved again.

\section{Experimental Section}

All reagents and solvents were reagent grade or were purified by standard methods before use and the reactions were routinely carried out under an inert atmosphere. Silica gel 60 (70-230 mesh ASTM) used for column chromatography was supplied by E. Merck. Analytical thin layer cluromatography (tlc) was performed on silica gel with fluorescent indicator coated on aluminium sheets. Melting points were taken using an Electrothermal melting point apparatus and are uncorrected. Microanalyses were obtained using a Carlo Erba EA 1180 element analyzer. Mass spectra were obtained using a ThermoQuest Polaris Q mass spectro- 
meter operating at $70 \mathrm{eV}$. Infrared spectra were recorded on a Nicolet Magna 550 FTIR spectrometer. The ${ }^{1} \mathrm{H}$ and ${ }^{13} \mathrm{C}$ NMR spectra were measured on a Gemini 300 spectrometer using deuterochloroform. All chemical shifts are reported in parts per million $(\delta)$ relative to tetramethylsilane.

4-Chloro-3-nitrobenzotrifluoride and 2-methylaziridine were purchased from Aldrich Chemical Company

4-(2-Methylaziridin-1-yl)-3-nitrobenzotrifluoride

To a solution of 2-methlaziridine $(3.71 \mathrm{~g} .65 \mathrm{mmol})$ and $\mathrm{Et}_{3} \mathrm{~N}(10.1 \mathrm{~g} .100 \mathrm{mmol})$ in $60 \mathrm{~mL}$ of THF was added 4chloro-3-nitrobenzotrifluoride (1.9.02 g. $40 \mathrm{mmol}$ ) and the mixture was stirred at $60^{\circ} \mathrm{C}$ for $48 \mathrm{~h}$. The solvent was removed on a rotavapor and the residue was partitioned between water $(20 \mathrm{~mL})$ and $\mathrm{CH}_{2} \mathrm{Cl}_{\Sigma}(100 \mathrm{~mL})$. The organic layer was removed after drying over $\mathrm{MgSO}_{4}$ and the residue was crystallized with hexane to give $9.16 \mathrm{~g}(93 \%)$ of $2 . \mathrm{mp}$ $65^{\circ} \mathrm{C}:$ IR $(\mathrm{KBr}) 1634,1565,1534,1328,1277,1153,1126$. $912.846 \mathrm{~cm}^{-1}$. ${ }^{1} \mathrm{H}$ NMR $\delta 1.45$ (d. $\left.3 \mathrm{H} . J=5.5 \mathrm{~Hz}\right) .2 .25(\mathrm{~d}$. lH. $J=6.1 \mathrm{~Hz}) .2 .32(\mathrm{~d} .1 \mathrm{H} . J=3.7 \mathrm{~Hz}) .2 .50(\mathrm{~m}, 1 \mathrm{H}) .7 .21$ (d. $1 \mathrm{H} . J=8.5 \mathrm{~Hz}$ ). 7.68 (d. $1 \mathrm{H} . J=8.5 \mathrm{~Hz}$ ). 8.22 (s. $1 \mathrm{H}$ ): MS (mz. \%) $246\left(\mathrm{M}^{-}, 7\right) .174$ (17). 145 (25). 127 (11). 105 (100).

And. Calcd for $\mathrm{C}_{10} \mathrm{H}_{0} \mathrm{~F}_{3} \mathrm{~N}_{2} \mathrm{O}_{2}:$ C. 48.79: H. 3.68: N. 11.38. Found: C. 48.51: H. 3.55: N. 11.20 .

3-Amino-4-(2-methylaziridin-1-yl)benzotrifluoride (3). To a stirred solution of $2(2.95 \mathrm{~g} .12 \mathrm{mmol})$ and $5 \%$ palladium on charcoal $(0.50 \mathrm{~g} .2 .4 \mathrm{mmol})$ in $40 \mathrm{~mL}$ of $\mathrm{MeOH}$ was added $98 \%$ hydrazine monohy'drate $(2.0 \mathrm{~g} .40$ $\mathrm{mmol}$ ) in $10 \mathrm{~mL}$ of $\mathrm{MeOH}$ dropwise manner over $1 \mathrm{~h}$ at r.t. The mixture was stirred for additional $1 \mathrm{~h}$ at r.t. and filtered over celite. The filtrate was evaporated in vacuo and the residue was partitioned between water $(10 \mathrm{~mL})$ and $\mathrm{CH}_{2} \mathrm{Cl}_{2}$ $(50 \mathrm{~mL})$. The organic layer was removed after drying over $\mathrm{MgSO}_{4}$ and the residue was crystallized with hexane to give $2.36 \mathrm{~g}(91 \%)$ of $3 . \mathrm{mp} 124-125^{\circ} \mathrm{C}$ : IR (KBr) 3285.3149 . $1619,1495,1436,1405,1335,1277,1203,1161,1110$. $1075,1013,889,823,792,722.702 \mathrm{~cm}^{-1}:{ }^{1} \mathrm{H}$ NMR $\delta 1.40$ (d. $3 \mathrm{H} . J=4.2 \mathrm{~Hz}) .2 .08-2.12(\mathrm{~m} .3 \mathrm{H}) .5 .52$ (s. $2 \mathrm{H}) .6 .81$ (d. $1 \mathrm{H} . J=8.2 \mathrm{~Hz}) .7 .10(\mathrm{~d} .1 \mathrm{H} . J=7.0 \mathrm{~Hz}) .7 .43(\mathrm{~s} .1 \mathrm{H}) . \mathrm{MS}$ $(m z . \%) 216\left(\mathrm{M}^{+} .22\right) .201$ (15). $187(100) .167(28)$.

Anal. Caled. for $\mathrm{C}_{10} \mathrm{H}_{11} \mathrm{~F}_{3} \mathrm{~N}_{2}$ : C. $55.55:$ H. 5.13: N. 12.96 . Found: C. 55.33: H. 4.88: N. 12.67.

4-(2-Methylaziridin-1-yl)-3-ureidobenzotrifluoride 4 . General Procedhue: To a stirred solution of $3(2.16 \mathrm{~g} .10$ mmol) in $20 \mathrm{~mL}$ of $\mathrm{CH}_{2} \mathrm{Cl}_{2}$ was added the isocyanate $(9.5$ mmol) in $10 \mathrm{~mL}$ of $\mathrm{CH}_{2} \mathrm{Cl}_{2}$ dropwise manner at r.t. After stirring for $2 \mathrm{~h}$ at ambient temperature. the solvent was removed on a rotavapor. The residue was crystallized from hexane to give 4.

The yield. physical and spectral data of $t$ are as follows:

4a: $90 \%$ mp $160-16{ }^{\circ} \mathrm{C}:{ }^{1} \mathrm{H}$ NMR $\delta 1.22$ (d. $1 \mathrm{H} . J=5.2$ Hz). $2.06($ d. $1 \mathrm{H}, J=3.7 \mathrm{~Hz}$ ). $2.10(\mathrm{~d} .1 \mathrm{H} . J=6.1 \mathrm{~Hz}) .2 .23$ (m. $1 \mathrm{H}), 6.89$ (d. $1 \mathrm{H} . J=8.2 \mathrm{~Hz}), 7.13-7.34(\mathrm{~m} .6 \mathrm{H}) .7 .68(\mathrm{~s}$. lH) 7.80 (s. $1 \mathrm{H}) .8 .29$ (s. $1 \mathrm{H}$ ).

Anal. Calcd for $\mathrm{C}_{17} \mathrm{H}_{16} \mathrm{~F}_{3} \mathrm{~N}_{3} \mathrm{O}:$ C. $60.88: \mathrm{H}, 4.81: \mathrm{N}$. 12.53. Found: C. $60.55:$ H. $4.75:$ N. 12.30 .

4b: $86 \%$. mp $138-139^{\circ} \mathrm{C}:{ }^{1} \mathrm{H}$ NMR $\delta 1.39$ (d. $3 \mathrm{H} . J=5.5$
Hz). 2.21 (d. $1 \mathrm{H} . J=3.4 \mathrm{~Hz}$ ). 2.39 (m. 2H). 6.97 (d. 1H. $J=$ $8.5 \mathrm{~Hz}$ ). $7.26-7.47$ (m. 5H), 7.74 (s. 1H), 8.21 (s. $1 \mathrm{H}$ ). 9.53 (s. 1H).

Anal. Calcd. for $\mathrm{C}_{17} \mathrm{H}_{1} \mathrm{ClF}_{3} \mathrm{~N}_{3} \mathrm{O}:$ C. 55.22: H. 4.09: N. 11.36. Found: C. 55.11: H. 3.82: N. 11.05

tc: $88 \%$ mp $139-140^{\circ} \mathrm{C}:{ }^{1} \mathrm{H}$ NMR $\delta 1.40$ (d. $3 \mathrm{H} . J=5.2$ Hz). 2.22 (s. $1 \mathrm{H}) .2 .40$ (m. 2H), 6.95-7.16 (m. $4 \mathrm{H}) .7 .41-$ 7.44 (m. 1H). 7.74 (s. 1H). 8.19-8.24 (m. 1H). $8.46(\mathrm{~s} .1 \mathrm{H})$. 9.60 (s. $1 \mathrm{H})$.

Anal. Calcd for $\mathrm{C}_{17} \mathrm{H}_{15} \mathrm{~F}_{4} \mathrm{~N}_{3} \mathrm{O}:$ C. 57.79: H. 4.28: N. 11.89. Found: C. 57.68: H. 4.07: N. 11.67.

4d: $90 \%$. mp $145-146^{\circ} \mathrm{C}$ : ${ }^{l} \mathrm{H}$ NMR $\delta 1.41$ (d. $3 \mathrm{H}, J=5.9$ Hz). 2.22 (d. $1 \mathrm{H} . J=3.4 \mathrm{~Hz}$ ). 2.38 (m. $2 \mathrm{H}$ ). 3.80 (s. $3 \mathrm{H}$ ). 6.87-7.01 (m. 3H). 7.40-7.46 (m. 3H), 7.78 (s. 1H). 8.10 (s. 1H). 9.24 (s. $1 \mathrm{H}$ )

Anal. Calcd for $\mathrm{C}_{18} \mathrm{H}_{18} \mathrm{~F}_{3} \mathrm{~N}_{3} \mathrm{O}_{2}:$ C. 59.17: H. 4.97: N. 11.50. Found: C. 58.81: H. 4.71: N. 11.22.

te: $91 \%$. mp $138-139^{\circ} \mathrm{C}:{ }^{1} \mathrm{H}$ NMR $\delta 1.35$ (d. $3 \mathrm{H} . J=4.9$ Hz). 2.13 (d. $1 \mathrm{H}, J=3.7 \mathrm{~Hz}$ ). $2.28-2.35(\mathrm{~m} .2 \mathrm{H}) .2 .89(\mathrm{~d}$. $3 \mathrm{H} . J=4.9 \mathrm{~Hz}$ ). 6.12 (br s. $1 \mathrm{H}$ ). 6.92 (d. $1 \mathrm{H} . J=8.2 \mathrm{~Hz}$ ). 7.38 (d. $1 \mathrm{H} . J=8.5 \mathrm{~Hz}$ ). 7.64 (s. $1 \mathrm{H}$ ). 9.12 (s. $1 \mathrm{H}$ )

Anal. Calcd for $\mathrm{C}_{12} \mathrm{H}_{14} \mathrm{~F}_{3} \mathrm{~N}_{3} \mathrm{O}:$ C. 52.75: H. 5.16: N. 15.38. Found: C. 52.60: H. 4.97: N. 15.05.

2,3-Dihydro- $1 H$-imidazo[1,2-a] benzimidazoles 8 and 12,13-Dihydro-5 $H$-benzimidazo $[2,3-b][1,3]$ benzodiazepine 9. General Procedure: To a stirred solution of the appropriate urea $4(2 \mathrm{mmol})$ in $50 \mathrm{~mL}$ of $\mathrm{CH}_{2} \mathrm{Cl}_{2}$ was added $\mathrm{Ph}_{3} \mathrm{P}$ $(1.80 \mathrm{~g} .6 .8 \mathrm{mmol}) . \mathrm{CCl}_{4}(1.4 \mathrm{~mL} .14 \mathrm{mmol})$, and $\mathrm{Et}_{3} \mathrm{~N}(0.9$ mL. $6.8 \mathrm{mmol}$ ). The mixture was heated to reflux temperature for 2-24 h. After cooling to room temperature the reaction mixture was partitioned between water $(20 \mathrm{~mL})$ and $\mathrm{CH}_{2} \mathrm{Cl}_{2}(50 \mathrm{~mL})$. The solvent was removed after drying over $\mathrm{MgSO}_{4}$ and the residue was chromatographed on silica gel colunun, eluted with hexane-EtOAc $4: 1$ to give the products 8 and 9 .

The reaction time, yield phsical and spectral data of 8 and 9 are as follows:

8a: 24 h. $70 \%, \mathrm{mp} 148-150^{\circ} \mathrm{C}$ : IR ( $\left.\mathrm{KBr}\right) 1611.1561$. 1530. 1499,1440,1374, 1320.1242.1161,1110 cm${ }^{-1}:{ }^{1} \mathrm{H}$ NMR $\delta 1.66($ d. $3 \mathrm{H}, J=6.4 \mathrm{~Hz}), 4.26(\mathrm{dd} .1 \mathrm{H} . J=15.6$ and $7.3 \mathrm{~Hz}$ ). 4.33 (dd. $\mathrm{lH} . J=15.6$ and $4.5 \mathrm{~Hz}$ ). $4.48(\mathrm{~m} . \mathrm{lH}$ ). 7.04-7.54 (m. 7H). 7.84 (s. $1 \mathrm{H}$ ): ${ }^{13} \mathrm{C}$ NMR $\delta 22.6 .50 .9,56.2$. $107.4,114.7 .117 .9 .118 .8 .124 .3 .124 .7,124.8 .\left(\mathrm{d}, J_{\mathrm{CF}}=262\right.$ Hz). 129.2, 136.0. 139.2, 141.7, 151.5: MS (mz. \%) 317 $\left(\mathrm{M}^{-}, 100\right) .316(35), 302(80) .276(20), 117(16)$.

Anal. Calcd for $\mathrm{C}_{17} \mathrm{H}_{14} \mathrm{~F}_{3} \mathrm{~N}_{3}: \mathrm{C}, 64.35: \mathrm{H}, 4.45: \mathrm{N}, 13.24$. Found: C. 64.10: H. 4.31: N. 13.02 .

9a: 24 h. $6 \%$. mp 166-168 ${ }^{\circ} \mathrm{C}: \mathrm{IR}(\mathrm{KBr}) 3409.1697 .1634$. 1561. 1495. 1436. 1320. $1114 \mathrm{~cm}^{-1}:{ }^{1} \mathrm{H}$ NMR $\delta 1.68$ (d. $3 \mathrm{H}$. $J=6.1 \mathrm{~Hz}$ ). 4.03 (dd. $1 \mathrm{H} . J=9.5$ and $5.8 \mathrm{~Hz}$ ). 4.60 (dd. $1 \mathrm{H}$. $J=9.5$ and $8.9 \mathrm{~Hz}) .4 .76(\mathrm{~m} .1 \mathrm{H}) .7 .04-7.44(\mathrm{~m} .4 \mathrm{H}) .7 .68-$ 7.71 (m. 2H). 7.81 (s. 1H): ${ }^{13} \mathrm{C}$ NMR $\delta 19.8 .48 .7,58.6$ $107.2 .114 .8 .115 .4 .115 .7,117.6,122.1 .123 .9,125.0\left(\mathrm{~d} . J_{\mathrm{CF}}\right.$ $=272 \mathrm{~Hz}), 129.2,129.4,133.0(\mathrm{C} 13 \mathrm{a}), 139.5,148.0 .157 .3 \mathrm{.}$ MS (mz, \%) $317\left(\mathrm{M}^{-}, 100\right) .302(95) .276(15) .213(10)$. Anal. Calcd for $\mathrm{C}_{17} \mathrm{H}_{14} \mathrm{~F}_{3} \mathrm{~N}_{3}:$ C. 64.35: H, 4.45: N, 13.24 . Found: C. 64.16: H. 4.09: N. 13.39 . 
8b: 24 h. $54 \%$. mp $155-156^{\circ} \mathrm{C}$ : IR (KBr) 1607.1549. 1526. 1487. 1444, 1413, 1328, 1254, 1168, 1126. $1056 \mathrm{~cm}^{-1}$ : ${ }^{l} \mathrm{H}$ NMR $\delta 1.69$ (d. $3 \mathrm{H} . J=6.7 \mathrm{~Hz}$ ). 4.26 (dd. $1 \mathrm{H} . J=15.9$ and $7.6 \mathrm{~Hz}$ ). 4.36 (dd $1 \mathrm{H} . J=15.9$ and $3.9 \mathrm{~Hz}) .4 .50(\mathrm{~m}$. 1H) $7.14-7.51(\mathrm{~m}, 6 \mathrm{H}) .7 .83(\mathrm{~s}, 1 \mathrm{H}):{ }^{13} \mathrm{C}$ NMR $\delta 22.8,51.1$. $56.5,107.7,114.9 .118 .1,120.2 .124 .5 .124 .8\left(\mathrm{~d} . J_{\mathrm{CF}}=272\right.$ Hz). $125.0,128.0,129.2,136.0,138.0,151.2$. MS (mz.\%) $353(37), 351\left(\mathrm{M}^{-}, 100\right) .338(15), 336(51), 301(18) .275$ (50), $226(15), 151(26)$.

And. Calcd. for $\mathrm{C}_{17} \mathrm{H}_{13} \mathrm{ClF}_{3} \mathrm{~N}_{3}:$ C. 58.05: H. 3.73: N. 11.95. Found: C. 57.75: H. 3.49: N. 11.71.

9b: 24 h. 8\%, mp 168-171 ${ }^{\circ} \mathrm{C}$ : IR (KBr) 3405. 1708, 1615 . 1553, 1495, 1324. $1165,1118 \mathrm{~cm}^{-1}$. ' $\mathrm{H}$ NMR $\delta 1.62$ (d. $3 \mathrm{H}$. $J=6.4 \mathrm{~Hz}$ ) 4.11 (dd. $1 \mathrm{H} . J=7.9$ and $5.9 \mathrm{~Hz}) .4 .27$ (dd. $1 \mathrm{H}$. $J=7.9$ and $5.5 \mathrm{~Hz}), 4.44(\mathrm{~m}, 1 \mathrm{H}), 6.99-7.67(\mathrm{~m}, 6 \mathrm{H}) \cdot{ }^{13} \mathrm{C}$ NMR $\delta 19.8,48.8,58.6,107.4,115.1,116.6,117.9 .118 .4$. $124.9\left(\mathrm{~d}, J_{\mathrm{CF}}=272 \mathrm{~Hz}\right), 127.0,127.5,129.2 .133 .0 .136 .8$ (C13a), 138.1 147.6, 147.8: MS (mz,\%) $353(32) .351\left(\mathrm{M}^{+}\right.$. 100). 338 (16). $336(52) .301$ (16). 275 (39). 151 (19).

And. Calcd for $\mathrm{C}_{17} \mathrm{H}_{13} \mathrm{ClF}_{3} \mathrm{~N}_{3}$ : C. 58.05: H. 3.73: N. 11.95. Found: C. 57.79: H. 3.50: N. 11.68 .

8c: 5 h. $77 \%$. mp $125-127^{\circ} \mathrm{C}$ : IR (KBr) 1619. 1553, 1518 . 1452, 1382, 1328, 1250, 1168, 1133, $1052 \mathrm{~cm}^{-1} .{ }^{1} \mathrm{H}$ NMR $\delta$ $1.70(\mathrm{~d} .3 \mathrm{H} . J=6.7 \mathrm{~Hz}) .4 .31(\mathrm{dd}, 1 \mathrm{H}, J=15.8$ and $7.9 \mathrm{~Hz})$. 4.36 (dd. $1 \mathrm{H} . J=15.8$ and $4.3 \mathrm{~Hz}$ ). $4.50(\mathrm{~m} . \mathrm{lH}) .7 .00-7.43$ (m. 5H). 7.87 (s. 1H). $8.37(\mathrm{~m} .1 \mathrm{H}) .{ }^{13} \mathrm{C}$ NMR $\delta 22.6 .51 .0$. $56.1,107.7 .114 .8,117.9 .119 .4,119.9,122.8,124.3,124.6$. 124.8. (d. $\left.J_{\mathrm{CF}}=272 \mathrm{~Hz}\right), 127.6,130.2 .135 .9,141.5,152.4$ (d. $\left.J_{C F}=247 \mathrm{~Hz}\right): \mathrm{MS}(m z, \%) 335\left(\mathrm{M}^{+} .100\right), 320(58) .294$ (15) $226(14)$.

And. Calcd. for $\mathrm{C}_{17} \mathrm{H}_{13} \mathrm{~F}_{4} \mathrm{~N}_{3}:$ C. $60.90, \mathrm{H}, 3.91:$ N. 12.53 . Found: C. $60.72:$ H. $3.78:$ N. 12.35 .

9c: 5 h. 4\%. oil: IR (KBr) 3378, 1728, 1642. 1557. 1499. 1332. $1157.1114 \mathrm{~cm}^{-1}$. ' $\mathrm{H}$ NMR $\delta 1.41$ (d. $\left.3 \mathrm{H}, J=6.4 \mathrm{~Hz}\right)$. $3.90(\mathrm{dd} .1 \mathrm{H} . J=9.5$ and $5.7 \mathrm{~Hz}), 4.42$ (dd $1 \mathrm{H} . J=9.5$ and $8.5 \mathrm{~Hz}) .5 .11(\mathrm{~m} .1 \mathrm{H}) .7 .01-7.47(\mathrm{~m} .5 \mathrm{H}) .7 .71(\mathrm{~s} .1 \mathrm{H}) \cdot{ }^{13} \mathrm{C}$ NMR $\delta 19.3,47.9 .61 .5,107.3,111.2,114.6,116.7,117.5$. $119.7 .123 .7\left(\mathrm{~d}, J_{\mathrm{CF}}=272 \mathrm{~Hz}\right) .125 .1,126.5 .127 .2 .134 .1$ (C13a) $147.6,156.2\left(\mathrm{~d}, J_{\mathrm{CF}}=248 \mathrm{~Hz}\right), 159.3 \mathrm{MS}(\mathrm{mz} . \%)$ $335\left(\mathrm{M}^{-}, 100\right) .320(50) .316(15) .294$ (11). 226 (13).

And. Calcd. for $\mathrm{C}_{17} \mathrm{H}_{13} \mathrm{~F}_{4} \mathrm{~N}_{3}$ : C. $60.90: \mathrm{H}, 3.91:$ N. 12.53 . Found: C. $60.63:$ H. $3.75:$ N. 12.20 .

8d: 24 h. $78 \%$. mp $157-158^{\circ} \mathrm{C}$ : IR (KBr) 1615. 1561. 1510. 1444. 1374, 1332, 1238, 1161, 1114. $1065.1040 \mathrm{~cm}^{-1}$ : ${ }^{l} \mathrm{H}$ NMR $\delta 1.67$ (d. $\left.3 \mathrm{H}, J=6.7 \mathrm{~Hz}\right), 3.81$ (s. $\left.3 \mathrm{H}\right), 4.26(\mathrm{dd}$. $1 \mathrm{H} . J=15.9$ and $7.6 \mathrm{~Hz}), 4.32(\mathrm{dd} .1 \mathrm{H} . J=15.9$ and $3.7 \mathrm{~Hz}$ ). $4.50(\mathrm{~m}, \mathrm{lH}), 6.91-6.94(\mathrm{~m}, 2 \mathrm{H}) .7 .12-7.15(\mathrm{~m}, 1 \mathrm{H}) .7 .36-$ $7.45(\mathrm{~m} .3 \mathrm{H}), 7.81$ (s. $1 \mathrm{H}):{ }^{13} \mathrm{C}$ NMR $\delta 22.7 .50 .8,55.5$. $55.9 .107 .4,114.5,117.6,121.8,124.2,124.7,124.9$ (d. $J_{C F}$ $=271 \mathrm{~Hz}), 132.3,136.3,141.8,152.7,156.1: \mathrm{MS}(m z, \%)$ $347\left(\mathrm{M}^{+} .100\right), 332(59), 147(35)$.

Anal. Calcd for $\mathrm{C}_{18} \mathrm{H}_{16} \mathrm{~F}_{3} \mathrm{~N}_{3} \mathrm{O}:$ C. 62.24: $\mathrm{H}, 4.64: \mathrm{N}$. 12.10. Found: C. 61.93: H. 4.78: N, 11.88 .

9d: 24 h, $8 \%$, oil: IR (KBr) $3400,1700,1634,1561,1514$. 1433, 1316, 1242,1153,1114, 1044 $\mathrm{cm}^{-1}$. ' $\mathrm{H}$ NMR $\delta 1.53$ (d. $3 \mathrm{H} . J=6.1 \mathrm{~Hz}$ ). 3.81 (s. $3 \mathrm{H}$ ). 3.90 (dd. $1 \mathrm{H} . J=9.5$ and $4.9 \mathrm{~Hz}$ ) 4.40 (dd. $1 \mathrm{H} . J=9.2$ and $8.8 \mathrm{~Hz}$ ) $4.98(\mathrm{~m}, 1 \mathrm{H}$ ). $6.96-7.33(\mathrm{~m}, 3 \mathrm{H}) .7 .54-7.61(\mathrm{~m}, 2 \mathrm{H}) .7 .74(\mathrm{~s} .1 \mathrm{H}):{ }^{13} \mathrm{C}$ NMR $\delta 19.3,47.6,55.6,59.9,107.0,114.7,117.4,119.7$. 120.6. 123.7. 124.1. 125.1 (d. $\left.J_{C F}=272 \mathrm{~Hz}\right) .131 .3 .134 .0$. 136.9 (C.13a). 147.8, 155.9. 158.8: MS (mz. \%) $347\left(\mathrm{M}^{-}\right.$. $100), 332(60), 281(35), 207(40) .147(35)$.

Anal. Calcd for $\mathrm{C}_{18} \mathrm{H}_{16} \mathrm{~F}_{3} \mathrm{~N}_{3} \mathrm{O}:$ C. 62.24: $\mathrm{H}, 4.64: \mathrm{N}$. 12.10. Found: C. 61.91: H. 4.40: N. 11.75

8e (HCl salt): 2 h. $74 \%$ mp $129-130^{\circ} \mathrm{C}$ : IR (KBr) 3196. 1631, 1607, 1588, 1448, 1413, 1378, 1316, 1285, 1141. 1110. $1048,873,803 \mathrm{~cm}^{-1}$. ${ }^{l} \mathrm{H}$ NMR $\delta 1.61$ (d. $3 \mathrm{H} . J=6.7$ Hz). 3.16 (s. $3 \mathrm{H}, J=4.9 \mathrm{~Hz}), 4.14(\mathrm{~m} .2 \mathrm{H}), 4.40(\mathrm{~m}, 1 \mathrm{H})$. 4.64 (br s. $1 \mathrm{H}$ ). 7.06 (d. $1 \mathrm{H}, J=8.2 \mathrm{~Hz}$ ). 7.32 (d. $1 \mathrm{H} . J=8.2$ Hz). 7.75 (s. $1 \mathrm{H}):{ }^{13} \mathrm{C}$ NMR $\delta 22.7,30.1 .50 .6 .55 .5 .107 .0$. $113.8,117.0,124.1,124.9\left(\mathrm{~d}, J_{C F}=271 \mathrm{~Hz}\right) .136 .9 .142 .2$, 156.4: MS $(m z, \%) 293(26), 291\left(\mathrm{M}^{+}, 79\right), 256(100) .228$ (29). $215(42), 187(41)$.

Anal. Calcd for $\mathrm{C}_{13} \mathrm{H}_{13} \mathrm{ClF}_{2} \mathrm{~N}_{3}:$ C. 49.41: H, 4.49: N. 14.41. Found: C. 49.11: H. 4.28: N, 14.14.

Acknowledgment. This work was supported by Korea Research Foundation Grant (KRF-2001-015-DP0335).

\section{References}

1. Jen1. T.: Bender. P.: Van Hoeven. H.: Dienel. B.: Love. B. J. Med. Chent. 1973, 16,407 .

2. Spasov, A. A. Chernitov, M. V.: Anisimova. V. A.; Kuzmento. T. A.; Osipova, M. M Khint -Furnt. Zh. 2000. 34, 48, Chem. Atstr. 2001. 134. 216795 .

3. Anisimova. V. A.: Levchenko. M. V: Kovalev. G. V:: Spasov. A A.: Dudehenko. G. P.: Tsibanev. A. V: Aleksandrova. E. A. Khim. -Form. Zh, 1988. 22, 1212; Chem. Abstr. 1989, 110, 51270 .

4. North. R. J; Day, A. R. J. Heterocycl Chem. 1969.6.655 and references cited therein.

5. Lee. K.-I.: Kang. S.-U. Tetrahedron Lett. 1995. 36. 2815.

6. Lee. K.-J.: Lim. I.S. J. Heterocycl. Chem. 2002. 39.975.

7. Intermolecular cycloaddition reaction of aziridine with carbodiimide: see. Baeg, J.-O.: Alper. H. J. Org. Chem. 1992, 57.157.

8. Appel. R.: Kleinstüch, R: Ziehn, K. D. Chent. Ber. 1971, 104. 1335. 